\title{
Meta
}

Journal des traducteurs

Translators' Journal

\section{Compte rendu du congrès en interprétation en milieu social tenu à Montréal du 22 au 26 mai 2001 L'interprétation en milieu social : la complexité d'une profession}

\section{Georges L. Bastin}

Volume 46, numéro 4, décembre 2001

URI : https://id.erudit.org/iderudit/001988ar

DOI : https://doi.org/10.7202/001988ar

Aller au sommaire du numéro

Éditeur(s)

Les Presses de l'Université de Montréal

ISSN

0026-0452 (imprimé)

1492-1421 (numérique)

Découvrir la revue

Citer ce document

Bastin, G. L. (2001). Compte rendu du congrès en interprétation en milieu social tenu à Montréal du 22 au 26 mai 2001 L'interprétation en milieu social : la

complexité d'une profession. Meta, 46(4), 751-753.

https://doi.org/10.7202/001988ar 


\section{Compte rendu du congrès en interprétation en milieu social tenu à Montréal du 22 au 26 mai 2001}

\section{L'interprétation en milieu social: la complexité d'une profession}

Geneva Park, 1995: L'interprétation en milieu social se donne un congrès Critical Link 1. Un succès, c'est l'éveil d'une profession, son entrée sur la scène internationale. Les interprètes communautaires du monde sortent de l'ombre, se définissent et se réunissent pour la première fois.

Vancouver, 1998: Critical Link 2. Ces mêmes interprètes, plus nombreux, réfléchissent, revendiquent et se positionnent. Ensemble, ils sentent leur force grandir et leurs problèmes s'amenuiser.

Montréal, 2001: La profession s'affirme, se spécialise et donne ses premiers fruits.

L'interprétation en milieu social: la complexité d'une profession, tel était le thème du $3^{\mathrm{e}}$ congrès international tenu à Montréal du 22 au 26 mai 2001 dernier. Le bilan statistique est éloquent: 1 jour de formation, 4 jours de délibérations, 400 participants d'une vingtaine de pays, 6 ateliers, 80 communications, 4 conférences plénières et 2 tables rondes.

L’objectif de ce $3^{e}$ maillon essentiel était explicitement exprimé dans le thème du congrès ainsi que dans le logo choisi : un contorsionniste. Objectif amplement atteint par la diversité des thèmes abordés (droits de la personne - dignité - éthique - reconnaissance professionnelle - équité de traitement pour les minorités), des domaines touchés (santé - santé mentale - judiciaire - police - immigration — scolaire - théâtre), des situations décrites (pays: Afrique du Sud, Brésil, Colombie, Irlande, Japon, Malaisie, Nouvelle-
Zélande, Venezuela, ... et environnement: hôpital — poste de police - salles d'audience - jury langues à faible diffusion, etc.), des moyens mis en ouvre (formation - accréditation - rationalisation - rentabilité (cost effectiveness)), des approches adoptées (linguistique - traductologique pragmatique - psychologique - sociologique et multidisciplinaire) et des acteurs étudiés (interprète - client - gestionnaire - fournisseur de service - autres professionnels).

Une nouveauté à Montréal: la veille du congrès, six ateliers de formation de trois heures tenus dans les domaines de la langue des signes, de la consécutive, du juridique et du téléphonique. 115 participants en tout se sont ainsi vu crédités d'un Certificat de formation continue octroyé par l'Université de Montréal.

Comme nous le disions, à Montréal, la profession s'est affirmée, spécialisée et a donné ses premiers fruits. En effet, les interprètes en milieu social commencent petit à petit à panser leurs plaies et à s'assumer comme professionnels au même titre que d'autres intervenants. Ils commencent aussi à effectuer des enquêtes sur le terrain, à mener des recherches empiriques et à se pencher sur des aspects très spécifiques de leur art. Finalement, l'interprétation communautaire ne se contente plus d'apprendre, elle commence à enseigner et contribue à enrichir le débat sur les langues, la communication et la traductologie.

Principaux apports de Montréal: par domaine, les points saillants des délibérations ont été les suivants:

1) Santé: Domaine prioritaire, la santé est le plus grand employeur, celui qui pose le plus de problèmes culturels, éthiques et de spécialisation, celui qui se prête le mieux à la recherche empirique. Il a été montré que les soins peuvent être améliorés grâce aux interprètes et que ceux-ci doivent prendre part aux efforts d'organisation plus rationnelle des services.

2) Judiciaire: Deuxième domaine en importance, il commence à bénéficier d'une vision de plus en plus réaliste des choses grâce à l'analyse des interventions de l'interprète: ajouts, interruptions, etc. Dans la formation, l'accent doit être mis sur le contenu juridique, l'éthique et les connaissances culturelles.

3) Langues à faible diffusion: Parmi celles-ci, la langue des signes fait l'objet de nombreuses recherches. À Montréal, la présence active des Sourds et de leurs interprètes s'est manifestée de façon notoire par de nombreuses communications, de nombreuses interventions et de nombreuses références. On constate un niveau 
de formation des interprètes de plus en plus élevé, jusqu’à la maîtrise; de même, un intérêt pour la formation d'interprètes sourds et la création de services d'interprètes sourds. Le respect des droits de la personne exige le traitement équitable des langues à faible diffusion, ce qui pourrait se traduire notamment par l'intégration des langues autochtones et des langues des signes aux projets de recherche en interprétation.

4) Analyse des échanges: De plus en plus, les discours et les échanges interprétés sont scrutés, voire décortiqués. Excellents exemples en santé et en judiciaire. Ces analyses, surtout lorsqu'elles sont multidisciplinaires, jettent une lumière nouvelle sur les prestations des interprètes et offrent un champ de recherche intéressant.

L'analyse interactionnelle, en particulier, débouche sur des modèles qui expliquent beaucoup mieux le travail des interprètes.

5) Accréditation: Divers exposés ont porté sur des systèmes établis et rodés (Australie, Europe et Canada), ainsi que sur des systèmes partiels ou en devenir.

L'enjeu est celui de la mesure des aptitudes par des tests avec des critères d'évaluation fondés sur la réalité professionnelle. Les interprètes réitèrent le besoin de normes unifiées (homogénéisées) comme au sein de l'Union européenne et réclament la participation du secteur public aux efforts d'accréditation. L'accréditation reste la clé de la qualité, de la reconnaissance, de la dignité.

6) Prise de conscience des gestionnaires: Plusieurs conférenciers ont montré l'intérêt de «former» les fournisseurs de services, de les sensibiliser à l'importance de participer à la formation de leurs interprètes. Avec pour arguments principaux: la qualité, le cadre juridique, les finances.

Les interprètes, quant à eux, se doivent d'aider les premiers à faire un examen de conscience, à repérer les erreurs du passé. Les aider à reconstruire les services et à les rendre rentables.

7) Prise de conscience des gouvernements: Les gouvernements sont responsables du retard pris par l'interprétation communautaire dans plusieurs pays. Leur rôle est incontournable en santé et en judiciaire, et le réseautage (networking) devient important pour accrô̂tre l'efficacité et la rationalité.

8) Reconnaissance professionnelle: Les mentalités changent. Certaines associations ont créé des comités ou des groupes de consultation pour l'intégration des interprètes communautaires. La méfiance et la mécon- naissance d'hier font place à l'ouverture et la solidarité. Il reste néanmoins énormément à faire car cette reconnaissance ne tombera pas du ciel; il appartient aux interprètes de s'affirmer et de faire valoir leurs compétences.

L'objection majeure à la reconnaissance par les ordres professionnels (au Canada notamment) est l'absence de diplômes universitaires parmi les interprètes. D'où le caractère indispensable d'une formation universitaire.

9) Visibilité de l'interprète: L'interprète assume sa présence, son rôle d'acteur social. On ne peut plus nier sa présence et plusieurs recherches s'efforcent de mettre en évidence son rôle actif. Il n'est plus seulement un relais, mais joue notamment un rôle de «coordination » des échanges. Il fait entendre sa voix et sentir son pouvoir. Agent culturel et social, il a cessé d'être une boîte vocale (voice box) et un simple transmetteur.

Les modèles interactionnels sont des modèles de visibilité par excellence

10) Formation: plus que jamais la formation, en particulier universitaire, est la clé de la qualité et de la reconnaissance. Révolu le temps des interprètes «amis-proches-parents» des clients! Une formation solide pour le respect des droits de la personne et au service de la justice et de la dignité humaine.

Des manuels et des guides à l'intention des interprètes voient le jour dans divers pays.

La formation doit viser non plus des niveaux abstraits de qualité mais avoir pour objectif que l'interprète soit pleinement fonctionnel. Les critères d'évaluation doivent être déterminés en fonction de cet objectif.

La formation doit aussi inclure le métalangage suffisant pour rendre l'interprète capable d'expliquer son exercice. L'autonomisation des étudiants devient indispensable. De même une méthode efficace et rapide de préparation thématique et terminologique. Les techniques de consécutive acquièrent un regain d'intérêt.

La formation utilise de plus en plus la simulation de conditions réelles

11) Éthique: Thème omniprésent. Les interprètes réclament des codes d'éthique et de comportement précis et adaptés, mais expriment le besoin d'aller au-delà de ces codes. L'éthique qui doit être présente dans toute formation, s'adresse aussi aux fournisseurs de services. Malgré de grands principes bien définis et généralement acceptés, l'éthique continue de susciter de grands débats comme en a témoigné la table ronde du congrès de Montréal.

12) Conditions de travail: Les revendications portent sur la rémunération, l'attitude des 
clients et des fournisseurs, la documentation, la protection et la santé.

13) Recherche: De plus en plus évoquée et représentée dans les conférences. On constate de moins en moins de "simples» descriptions de cas ou de situations particulières au profit d'études, d'enquêtes, de recherches empiriques, avec leur méthodologie et leurs résultats respectifs. Montréal s'est "spécialisé ». Cependant, de nombreuses recherches font encore cruellement défaut. Les mener à bien aiderait les interprètes à mieux convaincre les gestionnaires, les clients et ... eux-mêmes! Une approche féministe serait souhaitable.

\section{QUESTIONS POUR L'AVENIR}

Certaines questions suscitées par les communications méritent d'être reposées:

Pratique professionnelle: Le cercle vicieux de la formation - rémunération - reconnaissance; la question de la documentation préalable; la «syndicalisation» ou le renforcement des associations; la durée des prestations; la protection physique et mentale des interprètes.

Visibilité: Des recherches doivent approfondir le rôle joué par l'interprète et sa collaboration étroite avec d'autres professionnels.

Unicité-multiplicité de l'interprétation: Débat fondamental pour l'avenir de la profession, car il s'avère nécessaire à la fois de s'unir les uns aux autres et de se distinguer les uns des autres. Les recherches sur cette dualité doivent alimenter la formation: comment envisager les cursus? Un tronc commun avec différentes filières spécialisées?

Formation: Besoin d'une formation intégrée commune assortie d'une spécialisation selon les domaines.

Mise au point de cursus structurés dans les universités afin d'une part de mettre fin à la formation sur le tas et de l'autre d'élever le niveau de formation en vue d'atteindre la reconnaissance professionnelle. Importance de la formation continue.

Éducation des fournisseurs de services, des utilisateurs et des autres professionnels: Tous doivent apprendre à travailler en symbiose avec les interprètes pour accroître la qualité et le bon déroulement des prestations. Il y a beaucoup à apprendre de cette collaboration.

\section{RECOMMANDATIONS}

Deux recommandations ont été formulées par les participants :

1) Protéger les interprètes ayant été impliqués dans des situations de conflit;

2) Se solidariser avec le travail des ONG du type «Justice à l'étranger» (contre les emprisonnements sommaires d'étrangers).

Ajoutons encore que, à tout seigneur tout honneur, plus des deux tiers des présentations du congrès de Montréal ont été interprétées en français/ anglais et en cinq langues signées.

Les congressistes ont par ailleurs observé une minute de silence à la mémoire des interprètes blessés ou tués à la suite de leur prestation.

Le congrès s'est terminé par un rendez-vous: en juin 2004, Un maillon essentiel 4 se tiendra à Stockholm, en Suède.

Un ouvrage collectif comportant une bonne vingtaine d'articles issus des communications de Montréal sera publié au premier trimestre 2002 chez John Benjamins.

En guise de conclusion, quelques phrases-repères, des idées glanées au hasard des communications et livrées en vrac. Bien que sorties de leur contexte, elles n'en portent pas moins tout leur poids...

Il n'y a pas de petites langues; par contre il y en a des grandes!

Une formation solide au service de la justice et de la dignité humaine.

L'étranger est-il un handicapé linguistique?

A code of ethics keeps us together.

Language policy is not a luxury item!

Interpreting is interpreting, in any setting

Community interpreters or interpreters involved in their community?

We are too embarrassed to live but unwilling to die...

Who's going to pay for our dreams?

Georges L. BAstin Université de Montréal, Montréal, Canada 